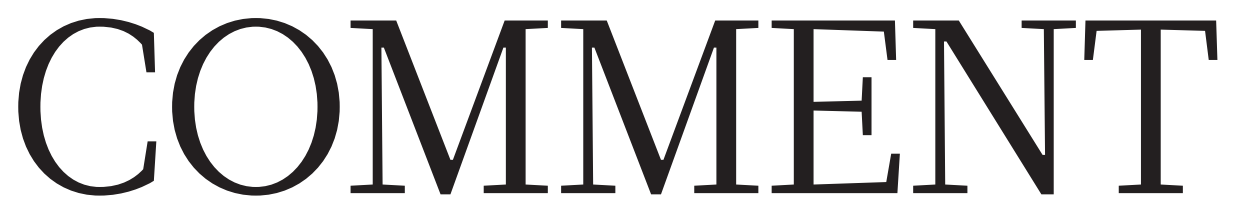

CHEMISTRY Let's raise a toast to the women of the periodic table $\mathbf{p . 5 5 9}$
HISTORY Ideas about elements evolved for 2,000 years before the atom's discovery p.563
LITERATURE Chemist Primo Levi's exquisite memoir, revisited $\mathbf{p . 5 6 4}$
CONSERVATION Madagascar's parks and forests need urgent protection $\mathbf{p . 5 6 7}$

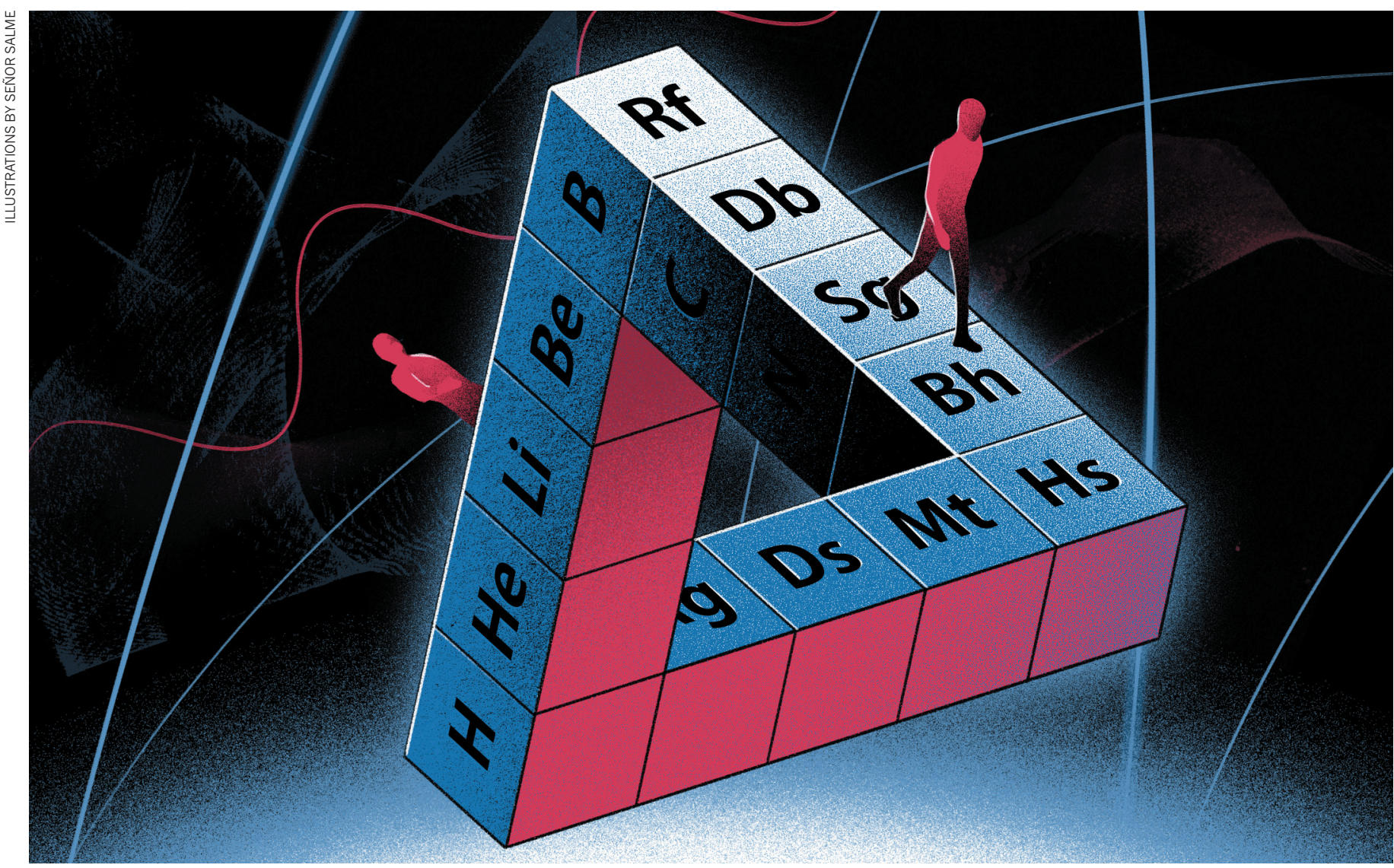

\title{
Can quantum ideas explain chemistry's greatest icon?
}

Simplistic assumptions about the periodic table lead us astray, warns Eric Scerri.

$\mathrm{S}$ uch has been the scientific and cultural impact of Dmitri Mendeleev's periodic table of the elements that many people assume it is essentially complete. In its 150th year, can researchers simply raise a toast to the table's many dividends, and occasionally incorporate another heavy synthetic element?

No - this invaluable compilation is still not settled. The placements of certain elements, even hydrogen and helium, are debated. Chemists dispute certain groupings, such as which elements should form group three of the periodic table (see go.nature. com/2vxnkqq). Traditionalists maintain that these comprise scandium, yttrium, lanthanum and actinium; a growing number thinks that lutetium and lawrencium should replace the last two on the basis of electronic structures. This matters, because moving an element to a different group might reveal new properties - it could become a candidate

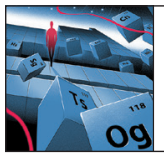

THE PERIODIC TABLE

A Nature special issue

go.nature.com/periodictable for inclusion in a high-temperature superconductor, for example.

The quantum-mechanical descriptions of electron arrangements in some atoms, notably transition elements such as copper and chromium, have been difficult to reconcile with the wider patterns of the periodic table. And it is unclear why there are more than 1,000 variants of the table, or whether there is one optimal version. Even the governing body of chemistry, the International Union of Pure and Applied Chemistry (IUPAC), is unsure. It claims not to back any particular 
$\checkmark$ arrangement ${ }^{1}$, yet the version on its website features a block of 30 elements below the main body of the table (see go.nature. $\mathrm{com} / 2 \mathrm{t} 2 \mathrm{uzmo}$ ). This is inconsistent with simple quantum-mechanical interpretations of atoms, which predict 28 such elements ${ }^{2}$.

Here I outline some of these inconsistencies, and explain how solving them continues to help physicists and chemists to understand and predict the behaviour of matter.

\section{PREDICTIVE POWER}

Mendeleev was not the first to try to arrange elements by their increasing order of atomic weight. He was the first to put such an arrangement to good use. His 1869 framework predicted the existence of several then-unknown elements, including gallium, germanium and scandium ${ }^{3}$. In the 150 years since, chemists have used it to predict atomic properties and have been inspired to perform landmark experiments. Physicists from J. J. Thomson to Erwin Schrödinger have used it as a test bed for theories of atomic structure and quantum mechanics.

Mendeleev did not know why elements had properties that recur periodically. Today, through many physicists' attempts to explain it, we know that atomic structure lies at the heart of the ordering of elements.

In the early twentieth century, physicists including Charles Glover Barkla and Ernest Rutherford noticed that the central charge of an atom is roughly half of its atomic weight. In 1911, a little-known Dutch economist and amateur scientist named Antonius van den Broek offered an explanation: atoms other than hydrogen are made up of multiples of 'alphons', a fundamental particle with half the mass of helium (two atomic mass units) and a single positive charge $e^{4}$.

Alphons have never been found, but van den Broek's hypothesis was the origin of the concept of atomic number - the number of protons in the nucleus of an atom (and thus the electrons around it) that determines an element's position in the periodic table. Physicist Henry Moseley confirmed this ordering in 1913 using X-ray spectroscopy ${ }^{5}$. This physical explanation justified previous ad hoc rearrangements of atoms in the periodic table, such as Mendeleev's switching of tellurium and iodine. (Iodine's atomic number is higher than that of tellerium, which has a higher atomic mass than iodine.)

As quantum mechanics developed in the 1920s, physicists Niels Bohr and Wolfgang Pauli developed a more sophisticated interpretation of the periodic table. The aufbau principle (from a German word meaning construction) describes the arrangements of electrons orbiting atomic nuclei, and is still taught today. Electrons orbit in a series of shells of increasing energy and distance from the nucleus (which are labelled with numbers); within each shell are orbitals of different types (s, p, d, f). The laws of quantum

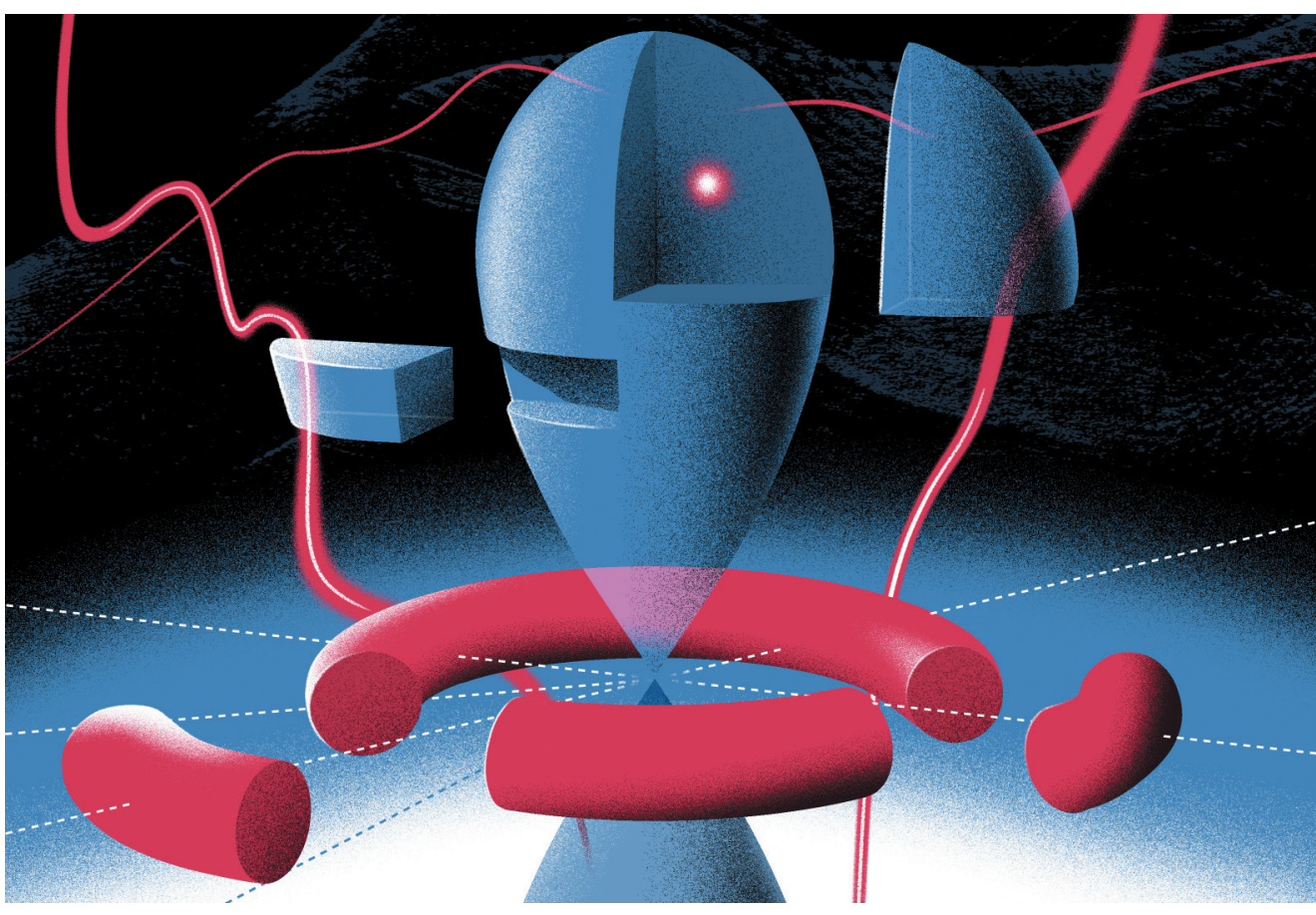

mechanics limit how many electrons can sit in each shell and orbital. Hydrogen has one electron in its 1s orbital; the next element, helium, has two. Lithium's third electron goes into the 2 s orbital, and so on.

The aufbau principle uses a simple numerical rule to describe the sequence in which orbitals are filled. This is known as the Madelung rule, after physicist Erwin Madelung, who (among others) formalized it in the 1930s. The sequence is straightforward for

"Quantum mechanics can explain these puzzling elements." the first three rows of the periodic table (in which elements have only $\mathrm{s}$ and $\mathrm{p}$ orbitals). The $3 p$ orbitals fill from aluminium to argon. But things get complicated in the fourth row. The $4 \mathrm{~s}$ orbital fills next, for potassium and calcium. But then the transition elements appear. The additional electron in the next element, scandium, doesn't go into 4p, but into $3 \mathrm{~d}$. Hence, transition metals are also known as d-block elements. The Madelung rule accommodates these non-intuitive steps, such that electron occupancy of $4 \mathrm{~s}$ precedes that of $3 \mathrm{~d}$, and $4 \mathrm{p}$ is occupied before $5 \mathrm{~s}$. But the Madelung rule has not yet been derived from quantum mechanics or other fundamental physical principles.

In 1969, on the 100th anniversary of the periodic table, chemist Per-Olov Löwdin declared this derivation to be one of chemistry's major theoretical challenges. It still is, 50 years on 6 .

\section{RULE BREAKERS}

Worse, there are 20 elements whose electron structures seem not to follow the Madelung rule. Some philosophers of science have argued that this points to a failure of quantum mechanics to explain the periodic table. I confess to having fallen into this trap myself ${ }^{7}$. Yet recent developments suggest that quantum mechanics can be reconciled with the aufbau principle and Madelung rule, if one looks deeper.

Chromium is one such anomalous element. The Madelung rule predicts that it should have four electrons in its $3 \mathrm{~d}$ orbitals and two in its 4 s orbital. However, spectroscopy of chromium reveals a different configuration: five electrons in the $3 \mathrm{~d}$ orbitals and one in its 4s. Similarly, copper, niobium, ruthenium, rhodium and a dozen other elements have one extra electron in their $\mathrm{d}$ or f orbitals, rather than in their outermost s orbitals as one might expect.

In 2006, theoretical chemist Eugen Schwarz and his colleagues moved the debate along ${ }^{8}$. According to the probabilistic approach of quantum mechanics, an atom can exist in a range of possible electronic configurations at the same time. For a given energy, there's a chance that an electron might lie in or across several orbitals. All of these options and their probabilities need to be considered when deriving the most stable configuration. After averaging, the predicted electronic states of most atoms agree with the Madelung rule. And the calculations predict the anomalous states correctly, in agreement with experiments.

Thus, quantum mechanics can explain these puzzling elements. However, most chemists, physicists and textbook writers are unaware of this.

In 2010, Schwarz and his team explained another quirk of transition metals ${ }^{9}$. The order in which electrons are released when some atoms are ionized also doesn't seem to 
follow the Madelung rule. Although scandium's extra electron lies in its 3d orbital, experiments show that, when it is ionized, it loses an electron from $4 \mathrm{~s}$ first. This doesn't make sense in energetic terms - textbooks say that 4 s should have lower energy than $3 \mathrm{~d}$. Again, this problem has largely been swept under the rug by researchers and educators.

Schwarz used precise experimental spectral data to argue that scandium's $3 \mathrm{~d}$ orbitals are, in fact, occupied before its $4 \mathrm{~s}$ orbital. Most people, other than atomic spectroscopists, had not realized this before. Chemistry educators still described the electronic structure of the previous element in the periodic table (calcium) carrying over into the next. In fact, each atom has its own unique ordering of energy levels. Scandium's 3d orbitals have lower energy than its 4 s orbital ${ }^{10}$. Schwarz urged chemists to abandon both the Madelung rule and Löwdin's challenge to derive it.

Schwarz is correct in saying that the Madelung rule is violated when it comes to the progressive occupation of orbitals in any particular atom. But it is still true that the electron that differentiates an element from the previous one in the table follows Madelung's rule. In the case of potassium and calcium, the 'new electron' relative to the previous atom is a 4 s electron. But in scandium, the electron that differentiates it from calcium is a $3 \mathrm{~d}$ one, even though it is not the final electron to enter the atom as it builds up.

In other words, the simple approach to using the aufbau principle and the Madelung rule remains valid for the periodic table viewed as a whole. It only breaks down when considering one specific atom and its occupation of orbitals and ionization energies.

The challenge of trying to derive the Madelung rule is back on.

\section{THEORIES STILL NEEDED}

This knowledge about electron orbitals does not change the order or placement of any elements in the table (even the anomalous 20 cases). It does enhance its theoretical underpinning. It shows how resilient the periodic table continues to be, along with the rules of thumb that have developed around it, such as the Madelung rule.

Quantum mechanics does a great job of explaining specific properties of atoms. Yet something more is needed to see the big picture. Although Schwarz cautions against superficial quantum-mechanical accounts of chemical facts, a deep dive into quantum mechanics might reveal a fundamental explanation of the Madelung rule, or a new way of thinking about it.

Even 150 years on, theoretical chemists, physicists and philosophers still need to step in to comprehend the gestalt of the periodic table and its underlying physical explanation. Experiments might shed new light, too, such as the 2017 finding that helium can form the compound $\mathrm{Na}_{2} \mathrm{He}$ at very high pressures $^{11}$. The greatest icon in chemistry deserves such attention.

Eric Scerri is a historian and philosopher of chemistry at the University of California, Los Angeles, California, USA.

e-mail:scerri@chem.ucla.edu

1. Leigh, G. J. Chem. Int. 31(1), 4-6 (2009).

2. Schaeffer, B. J. Mod. Phys. 5, 43128 (2014)

3. Scerri, E. R. The Periodic Table: Its Story and lts Significance 131-140 (Oxford Univ. Press, 2007)

4. Scerri, E. A Tale of Seven Scientists and a New Philosophy of Science 41-62 (Oxford Univ. Press, 2016).

5. Scerri, E. in For Science, King \& Country: The Life and Legacy of Henry Moseley (eds MacLeod, R., Egdell, R. G. \& Bruton, E.) 102-118 (Uniform, 2018).

6. Löwdin, P.-O. Int. J. Quantum Chem. S3, 331-334 (1969).

7. Scerri, E. R. in Essays in the Philosophy of Chemistry (eds Scerri, E. R. \& Fisher, G.) 125-143 (Oxford Univ. Press, 2016).

8. Wang, S. G., Qiu, Y. X., Fang, H. \& Schwarz, W. H. E. Chem. Eur. J. 12, 4101-4114 (2006).

9. Schwarz, W. H. E. \& Wang. S.-G. Int J. Quantum Chem. 110, 1455-1465 (2010)

10. Moore, C. E. Selected Tables of Atomic Spectra. A: Atomic Energy Levels - Second Edition (US Dept of Commerce, National Bureau of Standards, 1983).

11. Dong, X. et al. Nature Chem. 9, 440-445 (2017).

\section{The women behind the periodic table \\ Brigitte Van Tiggelen and Annette Lykknes spotlight female researchers who discovered elements and their properties.}

$\mathrm{T}$ he story of how dozens of elements were corralled into a periodic table reaches beyond one person and one point in time. Scientists classified and predicted elements before and after Dmitri Mendeleev's 1869 framework. And many more worked to find and explain these new substances. Noble gases, radioactivity, isotopes, subatomic particles and quantum mechanics were all unknown in the mid-nineteenth century.

Here we spotlight some of the women who revolutionized our understanding of the elements. Marie Curie is the most celebrated, for her double Nobel-prizewinning research on radioactivity and for discovering polonium and radium ${ }^{1}$. Stories of other women's roles are scarce. So, too, is an appreciation of the skills required, including tenacity and diligence in performing experiments, sifting through data and reassessing theories.

Proving the discovery of a new element is hard. The first step is finding unusual activity - chemical behaviour or physical properties that cannot be ascribed to known elements, such as unexplained radioactive emissions or spectroscopic lines. Then the element, or its compound, must be isolated in large enough quantities for it to be weighed, tested and used to convince others.

\section{SEARCH AND SORT}

Marie Curie wasn't looking for elements when she started her $\mathrm{PhD}$ on 'uranium rays' in 1897. She wanted to explore radioactivity,

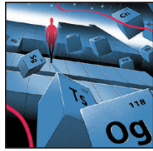

THE PERIODIC TABLE

A Nature special issue

go.nature.com/periodictable which had just been discovered by Henri Becquerel, in 1896. She came across pitchblende, an ore with radioactivity that was too strong to be explained by uranium alone. She suspected the presence of other elements, and brought in her husband, Pierre, to help.

In 1898, they identified spectroscopic lines of two new elements - radium and polonium. Yet it took them more than three years to grind, dissolve, boil, filter and crystallize tonnes of the mineral to extract just 0.1 gram of radium compound. (They struggled to do the same for polonium because of its short half-life.) Nobel prizes followed - the first shared by the pair and Becquerel in 1903 for discovering radioactivity, the second by Marie alone in 1911 for her discoveries of polonium and radium, and for the isolation and study of radium.

Positioning an element in the periodic 